\title{
CNTs-anchored egg shell membrane decorated with Ag-NPs as cheap but effective SERS substrates
}

\author{
Meiling Wang ${ }^{1}$, Guowen Meng ${ }^{1,2 *}$, Qing Huang ${ }^{3}$, Haibin Tang ${ }^{1}$, Zhongbo Li $^{1}$ and Zhuo Zhang ${ }^{1}$
}

\begin{abstract}
We report a simple approach for the fabrication of cheap but effective surface-enhanced Raman scattering (SERS) active substrates consisting of natural egg shell membrane (ESM) grafted with Ag-nanoparticles (Ag-NPs) decorated carbon nanotubes (CNTs) (denoted as Ag-NPs@CNTs@ESM), via ultrasonic adsorption of CNTs on the ESM and the subsequent Ag-sputtering. As high density Ag-NPs were sputtered onto each CNT anchored on the ESM nanofiber, homogeneous nanoscaled gaps between the neighboring Ag-NPs were achieved, leading to high SERS activity with excellent SERS signal homogeneity over the whole membrane. Using the Ag-NPs@CNTs@ ESM membranes as SERS-substrates, methyl parathion (one of the most hazardous insecticides), PCB-3 (one congener of polychlorinated biphenyls belonging to persistent organic pollutants) and bovine serum albumin (BSA, biomolecules) with a concentration down to $10^{-11} \mathrm{M}, 10^{-6} \mathrm{M}$ and $0.1 \mathrm{ppb}$ were detected respectively, showing promising potentials in rapid trace detection of environmental pollutants and biochemicals.
\end{abstract}

\section{INTRODUCTION}

Surface-enhanced Raman scattering or spectroscopy (SERS), as a nondestructive and powerful trace detection technique for target molecules, has been extensively explored for probing explosives, toxins and narcotics due to its high sensitivity, rapid response and fingerprint identification [1-5]. Silver nanostructures, with extraordinary localized surface plasmon resonance (LSPR), are widely employed as building blocks for effective SERS substrates with remarkable applications [6]. Great efforts have thus been devoted to the development of Ag-based SERS substrates. As three-dimensional (3D) SERS substrates can offer large surface area with high density of "hot spots", fabrication of 3D SERS-substrates has also aroused special attention. One strategy to develop 3D SERS-substrates is to combine Ag nanostructures with robust 3D-frameworks. Compared with the dispersed Ag colloidal nanoparticles (NPs), delicate spatial arrangement of Ag-NPs on the 3D-frameworks may ensure better reproducibility and reliability of SERS signals, and also provide additional signal enhancement due to the newly generated "hot spots" and possible cavity resonances [7-9]. Thus much effort has been made on the fabrication of SERS substrates with various arrangements of $\mathrm{Ag}$ nanostructures on nonplasmonic 3D-frameworks with high uniformity [6-17]. However, sophisticated fabrication procedures with high cost are usually required in the previously reported studies, which are time/energy-consuming. Therefore, it is still a challenge to achieve cost-effective Ag-nanostructure based 3D SERS substrates via facile methods. Furthermore, if natural material can be used as 3D-frameworks, it must be appealing.

For 3D SERS substrates, the major SERS effect stems from large surface area with large numbers of nanogaps known as "hot spots" [18]. Egg shell membrane (ESM), as a natural and green semi-permeable membrane, is mainly composed of interwoven and coalescing nanofibers, and thus intrinsically possesses large surface area. So ESM may be utilized as 3D-frameworks to load high density Ag-NPs for effective SERS substrates. However, the homogeneity for SERS signal can be critically affected by the irregular micropores in the interwoven fibers on the ESM. To achieve high uniformity and reproducibility of SERS signals, the microstructures of ESM should be properly modified. Carbon nanotubes (CNTs), due to their fascinating adsorbability to small molecules and simplicity in fabrication, were selected as the modification material for the ESM. Via ultrasonic adsorption, CNTs can be uniformly decorated onto the surface of each ESM nanofiber (denoted as CNTs@ESM membrane). Furthermore, via simple Ag-sputtering, zero dimensional (0D) Ag-NPs can be easily assembled onto the surface of the one-dimensional (1D) CNTs [18]. And these unique 0D@1D composite nanostructures can be beneficial to the enhancement of the local electromagnetic field and the corresponding SERS signal owing to the formation of abundant "hot spots" [18]. Furthermore, due to the excellent adsorbability of the CNTs to small molecules, target molecules can be readily captured by the CNTs@ESM

\footnotetext{
${ }^{1}$ Key Laboratory of Materials Physics, and Anhui Key Laboratory of Nanomaterials and Nanotechnology, Institute of Solid State Physics, Chinese Academy of Sciences, Hefei 230031, China

${ }^{2}$ University of Science and Technology of China, Hefei 230026, China

${ }^{3}$ Institute of Technical Biology, Hefei Institutes of Physical Science, Hefei 230031, China

"Corresponding author (email: gwmeng@issp.ac.cn)
} 
composite membrane, thus the SERS sensitivity would be significantly improved.

Herein, we present a simple and cheap approach for the fabrication of self-supported 3D SERS-active membranes of networked ESM grafted with Ag-NPs decorated-CNTs (denoted as Ag-NPs@CNTs@ESM), via a combinatorial process of ultrasonic adsorption of CNTs onto the surface of each ESM nanofiber and the subsequent Ag-sputtering, as shown schematically in Fig. 1. The assembled AgNPs on the outer surface of the CNTs are the key building blocks to achieve high SERS activity, which facilitate target molecules to be trapped onto the "hot spots" directly. The as-prepared Ag-NPs@CNTs@ESM membrane can provide not only a high density of "hot spots", but also a large surface area for adsorbing target molecules, ensuring the highly sensitive SERS detection of target analytes. The Ag-NPs@CNTs@ESM substrates were utilized for probing Rhodamine 6G (R6G), and a low detection limit of $1 \times 10^{-13}$ $\mathrm{M}$ has been achieved. To further demonstrate the practical applicability of the Ag-NPs@CNTs@ESM substrates for SERS detection of organic pollutants and biomolecules, methyl parathion (one of the most hazardous insecticides), PCB-3 (one congener of polychlorinated biphenyls belonging to highly toxic persistent organic pollutants) and bovine serum albumin (BSA, biomolecules) were used as the target molecules. And the Ag-NPs@CNTs@ESM substrates exhibited excellent sensitivity even for $10^{-11} \mathrm{M}$ methyl parathion, $10^{-6} \mathrm{M}$ PCB-3 and $0.1 \mathrm{ppb}$ BSA, showing promising potential in SERS-based trace detection of organic pollutants and biomolecules.

\section{EXPERIMENTAL DETAILS}

\section{Fabrication and characterization of the Ag-NPs@CNTs@ ESM 3D SERS substrates}

Briefly, the ESM was obtained by dissolving the egg shell with a $10 \%$ acetic acid solution, and washed with deionized (DI) water and ethanol for several times. Scanning electron microscopy (SEM) observation on the ESM membrane reveals interwoven $3 \mathrm{D}$ networked nanofibers with diameters of 500-1000 nm (Figs 2a-c). The obtained ESM was treated with ammonia (immersed in a 1:10 (v/v) ammonia spirit solution for $30 \mathrm{~min}$ ) before CNTs modification. 50 mg carboxylated CNTs with diameters of 30-50 nm were dispersed into $100 \mathrm{~mL}$ DI water and ultrasonicated for 60 min before use. Then the ammonia-treated ESM was put into the carboxylated CNTs solution and subjected to ultrasonication for $1 \mathrm{~min}$, resulting in a CNTs-decorated ESM membrane, as shown in the SEM image (Fig. 2d). The ultrasonic treatments induced the CNTs to uniformly adsorb onto the surface of the ESM nanofibers (denoted as CNTs@ ESM) due to the strong interactions between the carboxylated CNTs and the ammonia-treated ESM. Obviously, the surface area of the ESM was further enlarged after CNTs decoration. The thickness of the resulted CNTs@ESM membrane was about $20 \mu \mathrm{m}$ (see Fig. S1). All the above

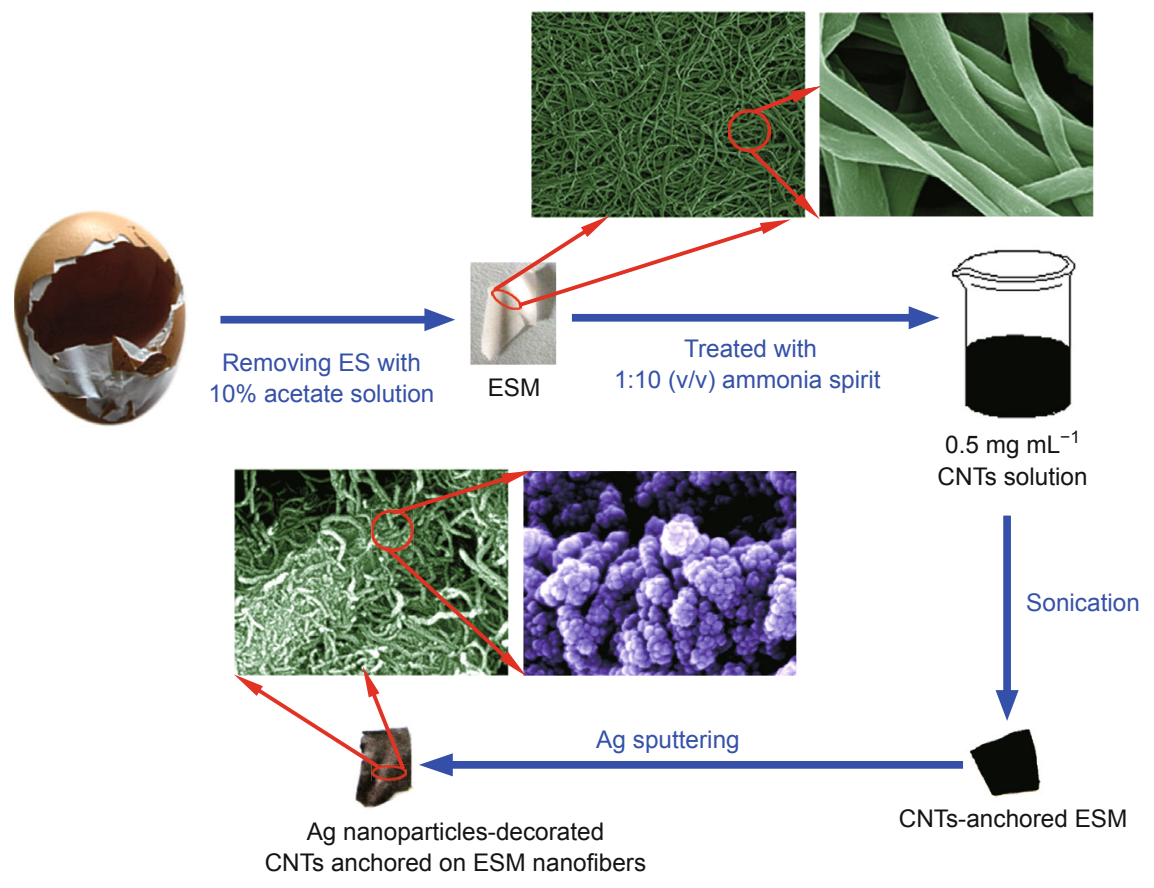

Figure 1 Scheme for the fabrication of the Ag-NPs@CNTs@ESM composite SERS substrate. 


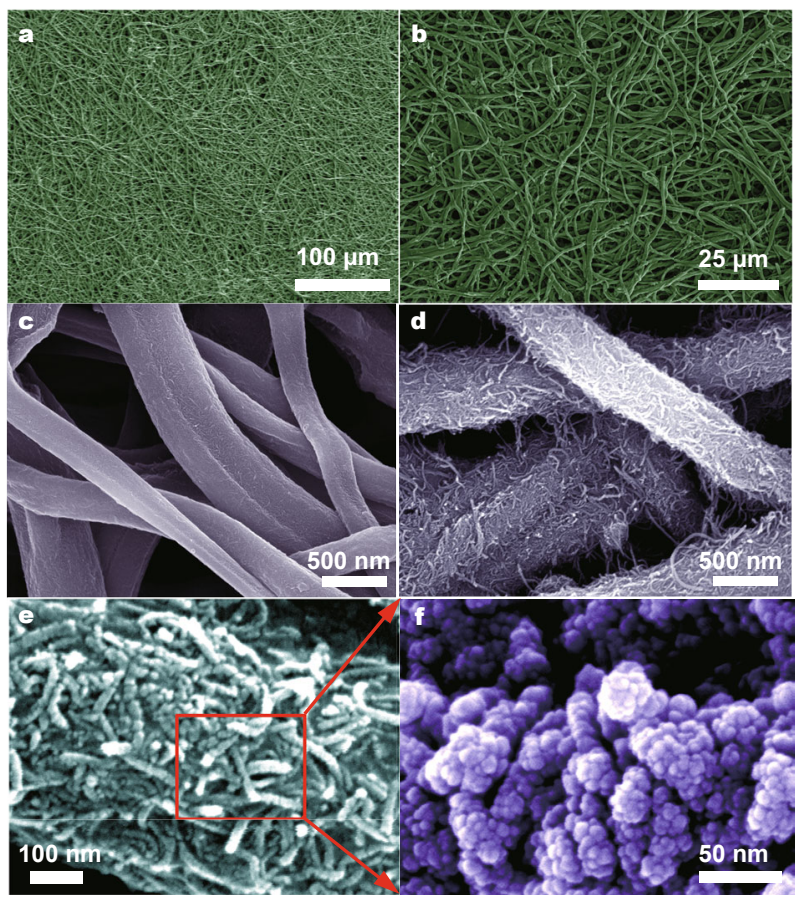

Figure 2 ( $a, b)$ SEM images of the ESM membrane at different magnifications; (c, d) SEM images of the ESM membrane before and after the decoration of CNTs at the same magnification; (e, f) SEM images of the Ag-NPs@CNTs@ESM substrate at different magnifications.

experiments were performed at ambient temperature.

As effective SERS substrates need abundant "hot spots", the CNTs@ESM membrane was subjected to Ag-sputtering to obtain nanogaps between the adjacent SERS-active Ag-NPs. It was observed that the ESM membranes with white color changed to black after being assembled with CNTs, and then became silvery after Ag-sputtering (shown in Fig. 1), confirming the formation of Ag-NPs@CNTs@ ESM. As shown in Figs 2e and f, for Ag-sputtering duration of $12 \mathrm{~min}, \mathrm{Ag}$-NPs of $\sim 10 \mathrm{~nm}$ in size densely and uniformly covered each CNT and each ESM nano fiber, so that nano-scaled gaps $(<10 \mathrm{~nm})$ between the adjacent Ag-NPs were formed, resulting in "hot spots" that can provide significant electromagnetic enhancement.

\section{Raman spectra measurements}

To estimate the SERS activity of the as-prepared Ag-NPs@ CNTs@ESM composite substrates, R6G was chosen as the target molecule. Firstly, the Ag-NPs@CNTs@ESM substrates were incubated in an aqueous solution containing different concentrations of R6G in the dark for $1 \mathrm{~h}$. Then the Raman spectra of dried R6G on the Ag-NPs@CNTs@ ESM composite substrates were measured using a confocal microprobe Raman spectrometer (Renishaw Invia, 532 $\mathrm{nm}$ laser line). All SERS spectra were acquired with a laser power of $0.1 \mathrm{~mW}$ and an acquisition time of $5 \mathrm{~s}$.

\section{RESULTS AND DISCUSSION}

\section{SERS activity evaluation of the Ag-NPs@CNTs@ESM}

As the networked Ag-NPs@CNTs@ESM composite substrates have porous structures with huge surface area, the probed target molecules can diffuse promptly onto the entire surface, which can reduce the response time and enhance the SERS signal intensity simultaneously. For control experiment, the SERS spectra of $10^{-8} \mathrm{M}$ R6G adsorbed on the Ag-sputtered ESM membrane with and without CNTs decoration (denoted as Ag-NPs@ESM) were collected (SEM image of the Ag-sputtered ESM membrane without CNTs decoration are given in Fig. S2), as shown in Fig. 3a, revealing that almost no SERS signal is observed for the ESM membrane without CNTs decoration compared with that of the Ag-NPs@CNTs@ESM. This result indicates that the CNTs modified on the ESM nanofibers make great contributions to the SERS efficiency of the composite substrates. In order to clarify this, we compared the SEM images of the Ag-NPs@CNTs@ESM and the Ag-NPs@ESM at the same magnification (Fig. S3), and it was proved that for the Ag-NPs@CNTs@ESM, uniform Ag-NPs of about 10 $\mathrm{nm}$ in size were formed, which gave rise to large quantities of nanogaps of sub-5 nm, while for the Ag-NPs@ESM, nonuniform Ag-NPs of about $20 \mathrm{~nm}$ in size were formed, resulting in nonuniform and large interparticle gaps. Therefore, the presence of the CNTs has greatly improved the uniformity of the Ag-NPs and reduced the interparticle gaps, which greatly improved the SERS activity of the substrates. Furthermore, the superior adsorption and collection capability of the CNTs (see Part S1 in the supplementary information) to small molecules also made great contributions to the outstanding SERS property of the AgNPs@CNTs@ESM.

To optimize the SERS activity of the Ag-NPs@CNTs@ ESM substrates, SERS spectra of $10^{-8}$ M R6G were measured using substrates with different Ag-sputtering durations from 4 to $18 \mathrm{~min}$ (sputtering current was all set at 40 $\mathrm{mA}$ ). As shown in Fig. 3b, the Raman activity is enhanced sharply with the sputtering duration increase from 4 to 12 min, while obviously decreased for longer Ag-sputtering duration. Variations of the SERS activity with different Ag-sputtering durations can be ascribed to the morphological evolution of the substrate. For short Ag-sputtering duration, i.e., 4 to $10 \mathrm{~min}$, only a few efficient "hot spots" (gaps between the adjacent Ag-NPs) were formed on the surface of the CNTs@ESM (see Fig. S5a), leading to a lower SERS activity; when the Ag-sputtering duration increases to 12 min, large quantities of "hot spots" are formed (Fig. $2 \mathrm{f}$ ), resulting in higher SERS signals. However, when the Ag-sputtering duration further increases, the SERS intensity decreases dramatically (Fig. 3b), being attributed to the 

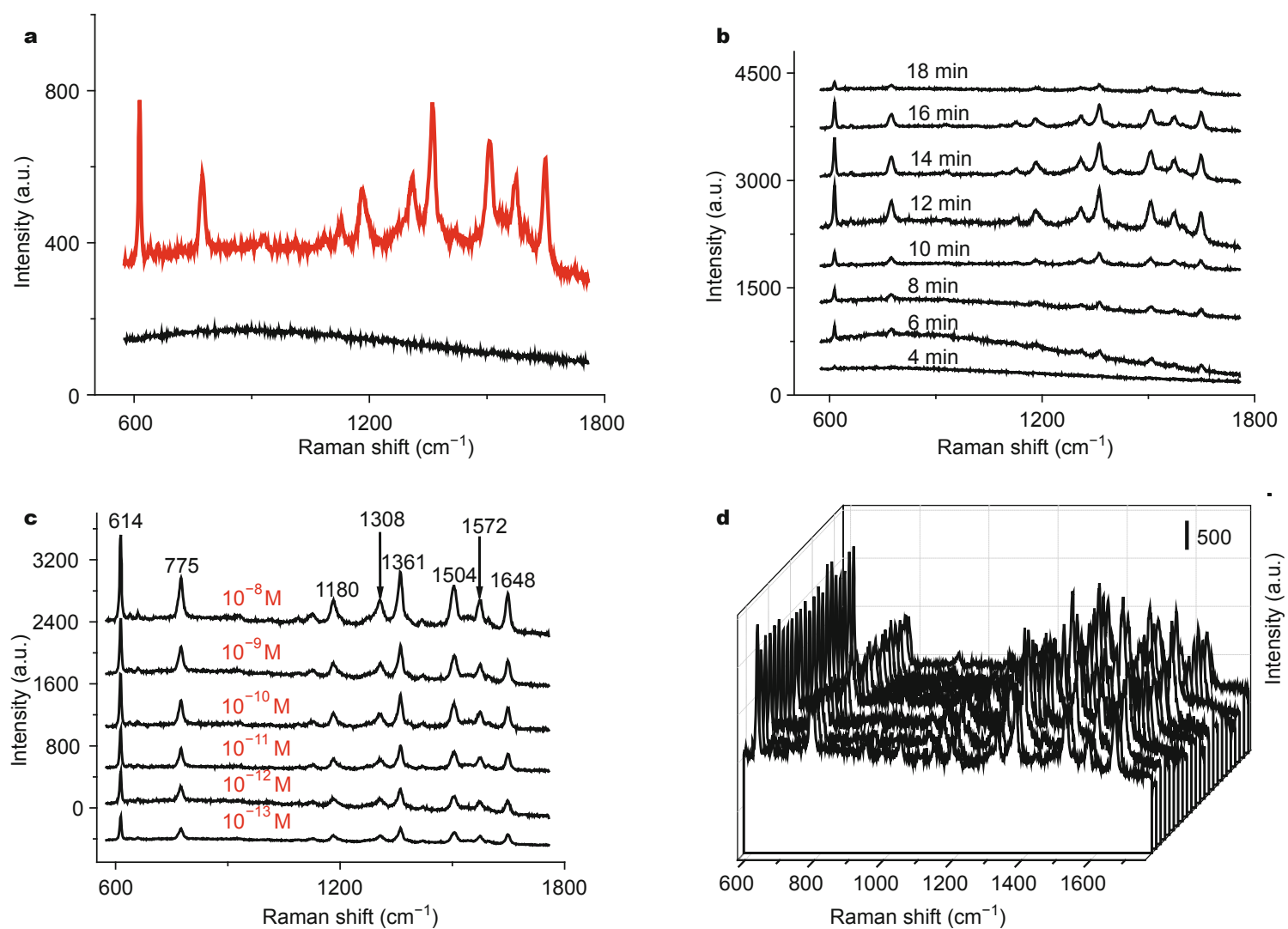

Figure 3 (a) The SERS spectra of 10-8 M R6G collected on the Ag-NPs@CNTs@ESM (red line) and Ag-NPs@ESM (black line) (Ag sputtering for $4 \mathrm{~min}$ ) respectively; (b) SERS spectra of $10^{-8} \mathrm{M}$ R6G collected on the substrates with Ag-sputtering for 4-18 min; (c) SERS spectra of different concentrations of R6G adsorbed on the Ag-NPs@CNTs@ESM substrate with Ag-sputtering for 12 min; (d) SERS spectra of 10 $0^{-8}$ M R6G at 23 randomly selected locations of the substrate.

fact that continuous sputtering of Ag results in the aggregation of the Ag-NPs and the disappearance of the gaps ("hot spots") between the adjacent Ag-NPs (see Fig. S5b). Thus, it is concluded that the Ag-NPs@CNTs@ESM with Ag-sputtering duration of 12 min shows the optimal SERS condition, which was therefore applied for SERS detection for the following work.

The SERS spectra of R6G with different concentrations adsorbed on the Ag-NPs@CNTs@ESM substrate with Ag-sputtering duration of $12 \mathrm{~min}$ are shown in Fig. $3 c$, revealing that Raman spectral feature of R6G is clearly recognized and the SERS signal intensity increases with the increase of R6G concentration. Even when the R6G concentration is down to $10^{-13} \mathrm{M}$, the R6G Raman characteristic bands can still be distinctly identified with a high signal-to-noise ratio, showing the high sensitivity of the as-prepared Ag-NPs@CNTs@ESM substrate.

The homogeneity and reproducibility of the SERS-active substrate are crucial to the SERS-based quantitative analysis. The homogeneity of our 3D SERS substrates over large areas was investigated by measuring the SERS spectra of $10^{-8} \mathrm{M}$ R6G at 23 randomly selected positions on the
Ag-NPs@CNTs@ESM membrane under identical Raman test conditions. As shown in Fig. 3d, the average relative standard deviation of the intensities (the peak at $614 \mathrm{~cm}^{-1}$ for example) is less than $20 \%$, revealing a good uniformity across the entire area of the SERS substrate. On the whole, the SERS activity and homogeneity are ascribed to the dense packing and the uniform distribution of the Ag-NPs on the surface of the CNTs@ESM membrane.

Furthermore, 4-aminothiophenol (4-ATP) was used to evaluate the average SERS enhancement factor (EF) of the Ag-NPs@CNTs@ESM substrates. The calculated EF is $1.25 \times 10^{7}$ (details can be found in Part S2 in the Supplementary information), and the high EF value of the Ag-NPs@ CNTs@ESM 3D substrates can be ascribed to the strong electromagnetic enhancement of the Ag-NPs located on the surface of the CNTs@ESM membrane [10]. In addition to the strong electromagnetic enhancement from the "hot spots" located on the same single CNT, our finite element analysis illustrates that electromagnetic enhancement induced by Ag-NPs on the neighboring CNTs also make contributions to the effective SERS signal of the as-prepared Ag-NPs@CNTs@ESM composite substrates (see Fig. S7). 
Taken together, the strong SERS effect is resulted from the electromagnetic enhancement due to the "hot spots" on the same CNT and the coupled electromagnetic enhancement from the neighboring Ag-NPs on different CNTs.

\section{SERS-based detection of methyl parathion, PCB-3 and BSA} using the Ag-NPs@CNTs@ESM substrate

For practical application, the Ag-NPs@CNTs@ESM was used as 3D SERS substrates for sensitive detection of methyl parathion, PCB-3 (4-chlorobiphenyl) and BSA. As shown in Fig. 4a, the spectral feature characteristics of methyl parathion can be clearly identified, and the methyl parathion molecules give a good signal-to-noise ratio even for a concentration as low as $10^{-11} \mathrm{M}$, further confirming the high SERS sensitivity of the Ag-NPs@CNTs@ESM as 3D SERS substrates for organic pollutants. The SERS spectra of different concentrations of PCB-3 are given in Fig. $4 \mathrm{~b}$, showing distinctive SERS peaks belonging to PCB-3, which correspond very well with the Raman spectrum of PCB-3 powder (black curve in Fig. 4b). And even for $10^{-6}$ M PCB-3, the crucial characteristic peaks of PCB-3 can still be distinguished, revealing that the Ag-NPs@CNTs@ESM as $3 \mathrm{D}$ SERS substrates have high sensitivity to PCB-3. Fur-
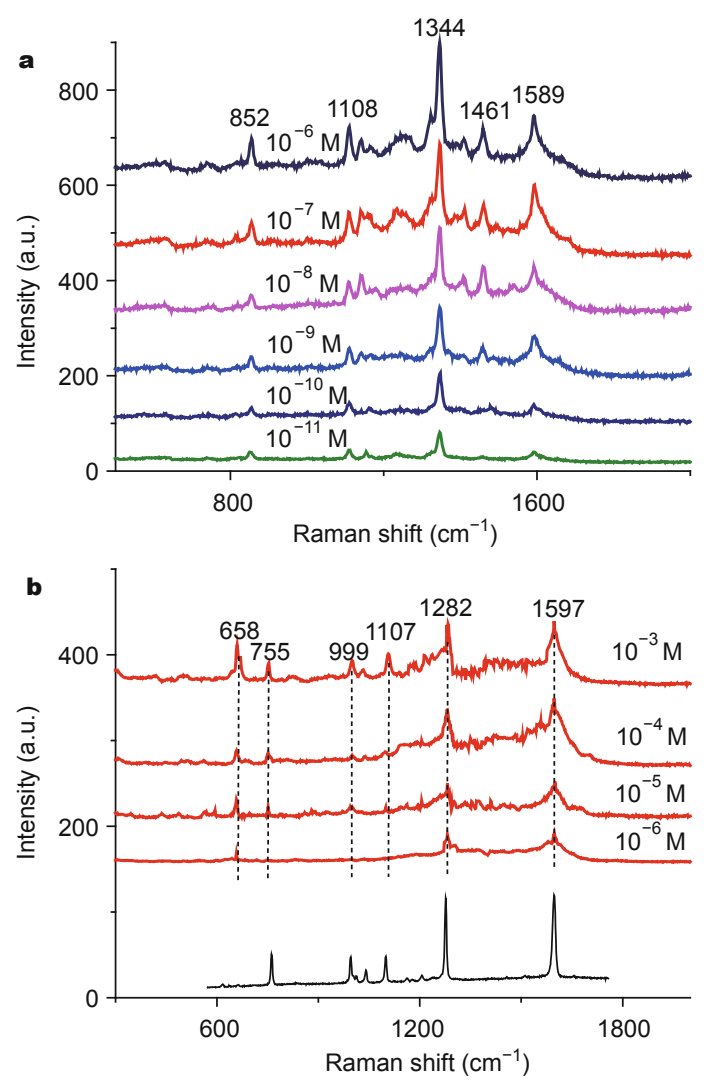

Figure 4 SERS spectra of (a) methyl parathion insecticide and (b) PCB3 at different concentrations with Ag-sputtering for 12 min. The black curve in (b) is the SERS spectrum of PCB-3 powder. thermore, the Ag-NPs@CNTs@ESM was also employed to probe BSA in solution, a protein widely used in biotic experiments, and the detection lower limit can reach 0.1 ppb (Fig. S8). As the major constitutes of the ESM is interwoven and coalescing protein, which is insoluble in most solvent, and is chemically inert at ambient temperature, the chemical stability of the ESM during the detection is excellent. Therefore, the environmentally friendly Ag-NPs@ CNTs@ESM, as a cheap but effective SERS substrate, has the promising potential to be used as a general assay for rapid trace detection of organic pollutants and chemical/ biological analytes.

\section{CONCLUSIONS}

In summary, we have presented a simple and cheap method to fabricate flexible and self-supported 3D SERS substrates consisting of Ag-NPs decorated-CNTs grafted on the nanofibers of the egg shell membrane. The 3D SERS substrates possess both abundant "hot spots" and a huge surface area to capture target molecules, which ensure their excellent SERS activity. For practical application, the 3D SERS substrates were utilized to probe R6G, methyl parathion, PCB3 and BSA, and low concentrations down to $10^{-13}, 10^{-11}$, $10^{-6} \mathrm{M}$ and $0.1 \mathrm{ppb}$ were reached respectively, showing the promising potential in SERS-based rapid detection of pollutants in the environments and biomolecules in medical fields. As effective 3D SERS substrates, the fabrication protocol of the Ag-NPs@CNTs@ESM is fairly simple, and especially the raw materials are low-priced and environmentally friendly, which is essential for mass production and extensive utilization. Such a design concept and fabrication strategy for the 3D substrates using natural ESM may open a new avenue to the building of 3D hybrid SERS substrates for other SERS-active noble metal nanostructures.

Received 4 January 2015; accepted 6 February 2015; published online 10 March 2015

1 Stiles P, Dieringer J, Shah N, Van Duyne R. Surface-enhanced Raman spectroscopy. Ann Rev Anal Chem, 2008, 1: 601-626

2 Jarvis R, Goodacre R. Characterisation and identification of bacteria using SERS. Chem Soc Rev, 2008, 37: 931-936

3 Ryder A. Surface enhanced Raman scattering for narcotic detection and applications to chemical biology. Curr Opin Chem Biol, 2005, 9: 489-493

4 Chou A, Jaatinen E, Buividas R, et al. SERS substrate for detection of explosives. Nanoscale, 2012, 4: 7419-7424

5 Zhu Y, Kuang H, Xu L, et al. Gold nanorod assembly based approach to toxin detection by SERS. J Mater Chem, 2012, 22: 2387-2391

6 Li Z, Meng G, Huang Q, et al. Galvanic-cell-induced growth of Ag nanosheet-assembled structures as sensitive and reproducible SERS substrates. Chem Eur J, 2012, 18: 14948-14953

7 Yilmaz M, Senlik E, Biskin E, et al. Combining 3-D plasmonic gold nanorod arrays with colloidal nanoparticles as a versatile concept for reliable, sensitive, and selective molecular detection by SERS. Phys Chem Chem Phys, 2014, 16: 5563-5570 
8 Wei W, Chen K, Ge G. Strongly coupled nanorod vertical arrays for plasmonic sensing. Adv Mater, 2013, 25: 3863-3868

9 Doherty M, Murphy A, Mc Phillips J, Pollard R, Dawson P. Wavelength dependence of Raman enhancement from gold nanorod arrays: quantitative experiment and modeling of a hot spot dominated system. J Phys Chem C, 2010, 114: 19913-19919

10 Wang X, Meng G, Zhu C, et al. A generic synthetic approach to large-scale pristine-graphene/metal-nanoparticles hybrids. Adv Funct Mater, 2013, 23: 5771-5777

11 QianY, Meng G, Huang Q, et al. Flexible membranes of Agnanosheet-grafted polyamide-nanofibers as effective 3D SERS substrates. Nanoscale, 2014, 6: 4781-4788

12 Tang $\mathrm{H}$, Meng G, Huang Q, et al. Arrays of cone-shaped $\mathrm{ZnO}$ nanorods decorated with $\mathrm{Ag}$ nanoparticles as 3D surface-enhanced Raman scattering substrates for rapid detection of trace polychlorinated biphenyls. Adv Funct Mater, 2012, 22: 218-224

13 Zhu C, Meng G, Huang Q, et al. Ag nanosheet-assembled microhemispheres as effective SERS substrates. Chem Commun, 2011, 47: 2709-2711

14 Zhu C, Meng G, Huang Q, Huang Z, Chu Z. Au hierarchical micro/ nanotower arrays and their improved SERS effect by Ag nanoparticle decoration. Cryst Growth Des, 2011, 11: 748-752

15 Zhu C, Meng G, Huang Q, et al. Large-scale well-separated Ag nanosheet-assembled micro-hemispheres modified with HS- $\beta$-CD as effective SERS substrates for trace detection of PCBs. J Mater Chem, 2012, 22: 2271-2278
16 Zhu C, Meng G, Huang Q, Huang Z. Vertically aligned Ag nanoplate-assembled film as a sensitive and reproducible SERS substrates for the detection of PCB-77. J Hazard Mater, 2012, 211: 389-395

17 Zhu C, Huang Q, Zhang Y, et al. Ostwald-ripening-induced growth of parallel face-exposed Ag nanoplates on micro-hemispheres for high SERS activity. Chem Eur J, 2013, 19: 9211-9217

18 Sun Y, Liu K, Miao J, et al. Highly sensitive surface-enhanced Raman scattering substrate made from superaligned carbon nanotubes. Nano Lett, 2010, 10: 1747-1753

Acknowledgements This work was supported by the National Key Basic Research Program of China (2013CB934304), the National Natural Science Foundation of China (21307138, 11274312, 11175204 and 51472245), the State Administration of Foreign Experts Affairs of Chinese Academy International Partnership Program for Creative Research Teams, and the President Foundation of Hefei Institutes of Physical Science, Chinese Academy of Sciences (YZJJ201312).

Author contributions Wang M designed the research work, performed the experiments and the data analysis; Wang M, Huang Q, Li Z, Tang $\mathrm{H}$ and Meng $\mathrm{G}$ wrote the paper. All authors contributed to the general discussion.

Conflict of interest The authors declare that they have no conflict of interest.

Supplementary information Supporting data are available in the online version of the paper.

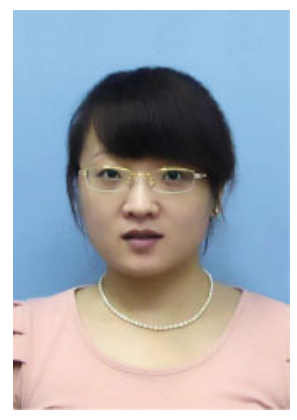

Meiling Wang was born in 1985. She received her PhD degree in Material Physics and Chemistry from the Institute of Solid State Physics (ISSP), Chinese Academy of Sciences (CAS), in 2012. Currently, she is a research associate at the ISSP, CAS. Her scientific research focuses on trace detection of heavy metal ions and persistent organic pollutants, and her research interests include nanomaterials and fluorescence sensors.

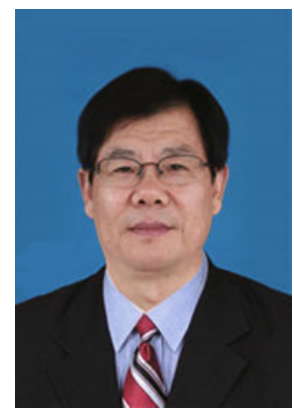

Guowen Meng was born in 1960. He received his BSc (1984), MSc (1987), and PhD degrees (1996) in Materials Science and Engineering, from Northwestern Polytechnic University, Xi'an, China. He has been working at the Institute of Solid State Physics, Chinese Academy of Sciences since 1996. He visited Rensselaer Polytechnic Institute (USA) from 2002 to 2004. His current research focuses on rational building of nanostructure arrays as platform for rapid detection of toxic pollutants.

中文摘要 本文报道了一种利用具有均匀纤维网络结构的鸡蛋膜作为三维骨架, 制备碳纳米管/鸡蛋膜复合表面增强Raman散射 (SERS) 祄底的简单廉价的方法. 首先, 借助超声吸附, 将碳纳米管组装到经过前处理的鸡蛋膜纤维的表面, 得到均匀的碳纳米管/鸡蛋膜复合 膜; 然后, 借助溅射方法, 将银纳米颗粒组装到碳纳米管和鸡蛋膜纤维的表面, 得到银纳米颗粒修饰的碳纳米管/鸡蛋膜复合膜. 这种方 法制备的复合膜表面的碳纳米管对小分子目标分析物有很好的吸附和富集作用; 同时，由于高密度银纳米颗粒的存在，衬底表面有大 量的SERS活性位点, 因此这种复合膜是一种有效的SERS祄底. 研究表明, 采用这种银纳米颗粒修饰的碳纳米管/鸡蛋膜复合SERS祄底, 可实现对农药甲基对硫磷、多氯联苯以及生物分子牛血清蛋白等的快速痕量识别. 由于该祄底的制备方法简单、原材料环保且来源 广泛、祄底的活性高, 所以该祄底在基于SERS技术对环境污染物和生物分子的快速痕量检测方面具有广泛的应用前景. 